\title{
Prepartal infection of the placenta with Neisseria gonorrhoeae
}

\author{
F YVERT,* E FROST,* P WALTER, † R GASS, $\neq$ AND B IVANOFF* \\ From the ${ }^{*}$ Laboratoire de Microbiologie, Centre International de Recherches Médicales de Franceville, \\ Franceville, the +Laboratoire de Pathologie, Centre Universitaire des Sciences de la Santé, Libreville, and \\ ¥Service de Gynécologie, Hôpital Provincial de Franceville, Franceville, Gabon
}

SUMMARY Placentas from 191 consecutive deliveries were cultured for Neisseria gonorrhoeae and other bacteria before being examined histologically. $N$ gonorrhoeae was seen on direct microscopical examination and cultured from two placentas, one of which had chorioamnionitis and the other had a granulocytic invasion of the membranes. In both patients, rupture of the membranes had occurred two hours or less before delivery, and thus after infection. A third patient was also found to have light gonococcal infection of the placenta. Six infections with group B streptococci were identified, but only one correlated with chorioamnionitis. Chorioamnionitis was observed in $16(8 \cdot 4 \%)$ and granulocytic infiltration of the membranes in $18(9 \cdot 4 \%)$ of the deliveries.

\section{Introduction}

Neisseria gonorrhoeae is a common pathogen of the urogenital tract, which is capable of causing local and systemic infections. Gonococcal pelvic inflammatory disease is rare in pregnant women, ${ }^{1}$ but gonorrhoea has occasionally been associated with the amniotic infection syndrome that includes prolonged rupture of the fetal membranes, premature deliveries, and chorioamnionitis. ${ }^{23}$ It has not been clear, however, whether such infection preceded (and was, or was not, responsible for) or followed rupture of the membranes. Prepartal infections could weaken the chorioamniotic membrane and, in severe cases, cause premature rupture.

In Franceville, Gabon chorioamnionitis has been reported in about $6 \%$ of births (Walter $P$ and Philippe E, unpublished observation) and $N$ gonorrhoeae has been cultured from the cervix of $5.5 \%$ of pregnant women. ${ }^{4}$ We therefore looked for possible in utero gonococcal infections and report here two cases of probable prepartal infection of the placenta with $N$ gonorrhoeae.

Patients, materials, and methods

We obtained samples from 191 consecutive patients at the time of delivery. On their arrival at the hospital,

Address for reprints: Dr B Ivanoff, Directeur Général, Centre International de Recherches Médicales de Franceville, BP 769, Franceville, Gabon

Accepted for publication 11 July 1984 cultures and slides were made from cervical secretions. After delivery, material from the fetal surface of the placenta near the umbilical cord was examined by microscopy and samples taken for culture. Swabs were inserted into a semisolid transport medium (TGV (transport des germes vivants) aerobie; Institut Pasteur Production (IPP), Paris). Within 24 hours they were streaked on selective and non-selective media, incubated in $10 \%$ carbon dioxide at $37^{\circ} \mathrm{C}$, and observed daily for three days. Oxidase positive colonies were analysed as described previously with neisseria identification galleries (IPP).$^{4}$ One slide was stained with Giemsa and another by Gram's method.

Histological observations were made on placental material prepared as reported previously. ${ }^{5}$ In addition, three samples of membrane were taken from each placenta: one from the area of rupture of the amniotic membrane nearest to the margin of the placenta, one from the placental margin nearest to the point of rupture, and one from the placenta close to the umbilical cord. Although granulocytic infiltration of the placental membranes, whether heavy or light, is currently considered to result from infection, we distinguished two forms: (a) granulocytic infiltration of the membranes, which we defined as inflammatory lesions restricted to the placental chorion layers close to the maternal side and without chorionic vasculitis; and (b) chorioamnionitis, which we defined as granulocytic infiltration of the whole thickness of the placental chorion, usually associated with chorionic vasculitis. Granulocytic infiltration 
showed few granulocytes (not more than 20 per high power field (x 400)), which exceeded 50 per high power field in chorioamnionitis.

\section{Results}

CASE 1

A woman aged 24 who delivered her fifth live child at term had presented with a vaginal discharge 17 days before delivery and was treated with pessaries containing neomycin, polymyxin B, nystatin, and acetarsol. Cultures for $N$ gonorrhoeae had proved positive, but the woman could not be located for further treatment until she arrived at hospital in labour. Rupture of the membranes occurred two hours before the delivery of a normal boy child (Apgar score 10 at one minute) weighing $2850 \mathrm{~g}$ and a placenta weighing $410 \mathrm{~g}$.

Histological examination of placental tissue showed a granulocytic infiltration of the chorionic membrane. $N$ gonorrhoeae was isolated in pure culture from the placenta and with other organisms from the cervical swab. The Gram stained slide made direct from the placenta showed numerous foci of both intracellular and extracellular Gram negative diplococci. Neither mother nor child was feverish. The child developed conjunctivitis on the following day and Chlamydia trachomatis was isolated, but not $N$ gonorrhoeae. C trachomatis was isolated subsequently from the mother.

CASE 2

A woman aged 23 and pregnant for a fifth time (after three live births and an abortion) was delivered at term of a girl child weighing $3070 \mathrm{~g}$ and a placenta weighing $550 \mathrm{~g}$. The membranes had been ruptured artificially half an hour before delivery. The child had an Apgar score of 10 at one minute and neither she nor the mother was feverish or had conjunctivitis during their three subsequent days in hospital. $N$ gonorrhoeae was not isolated from the vaginal swab, but culture of the placental sample showed a few colonies in pure form on selective media. Direct examination of the placental scraping also showed a few Gram negative diplococci. Histological examination of the placenta showed chorioamnionitis including granulocytic infiltration of the placental amnios. This infiltration was more dense in the extraplacental than in the placental amnios. The beginning of granulocytic invasion of the wall of one of the umbilical arteries was also noted.

\section{CASE 3}

A girl aged 15 gave birth at term to a $2660 \mathrm{~g}$ girl infant with a $630 \mathrm{~g}$ placenta after artificial rupture of the membranes one hour before delivery. The child was normal at birth and neither she nor the mother showed signs of fever during the following three days. $N$ gonorrhoeae was isolated from the cervical swab and four colonies in pure culture were isolated from the placental swab. No bacteria were apparent in the Gram stained smear made direct from the placenta. Examination of the placenta showed normal membranes without inflamation but some modification of the placenta caused by malaria. ${ }^{6}$

\section{Results}

The table summarises the histological and bacteriological results for the 191 deliveries. In $157(82 \cdot 2 \%)$ instances the placental membranes appeared to be normal, but $18(9 \cdot 4 \%)$ showed granulocytic infiltrations of the membranes and a further $16(8 \cdot 4 \%)$ had chorioamnionitis. Bacteriological examination showed heavy contamination with a mixed flora in 32 $(16 \cdot 8 \%)$ deliveries, probably because those placentas had been expelled with the fetal surface folded out rather than in. No bacteria were isolated from most (121) of the placental samples. In addition to the three placentas yielding gonococci described above, group B streptococci were isolated from six, but only one of these showed pathological change in the placental membranes.

In a parallel study, cultures were also taken from

TABLE Histological and bacteriological analysis of 191 placentas and bacteriological analysis of cultures from 16 newborn babies

No of babies with ophthalmia No of placentas yielding: neonatorum yielding:

\begin{tabular}{|c|c|c|c|c|c|c|c|c|c|}
\hline \multirow[b]{3}{*}{$\begin{array}{l}\text { Histology of } \\
\text { membranes }\end{array}$} & & & & & & & \\
\hline & \multirow[b]{2}{*}{$\begin{array}{l}\text { Mixed } \\
\text { bacteria }\end{array}$} & \multirow[b]{2}{*}{ Nil } & \multicolumn{2}{|l|}{ Gonococci } & \multicolumn{2}{|l|}{ Streptococci } & \multirow[b]{2}{*}{ Chlamydiae } & \multirow[b]{2}{*}{ Neisseriae } & \multirow[b]{2}{*}{ Other } \\
\hline & & & $\begin{array}{l}\text { Visible on } \\
\text { microscopy }\end{array}$ & $\begin{array}{l}\text { Not } \\
\text { visible on } \\
\text { microscopy }\end{array}$ & $\begin{array}{l}\text { Visible on } \\
\text { microscopy }\end{array}$ & $\begin{array}{l}\text { Not } \\
\text { visible on } \\
\text { microscopy }\end{array}$ & & & \\
\hline $\begin{array}{l}\text { Normal } \\
\text { Granulocytic }\end{array}$ & 30 & 121 & 0 & 1 & 3 & 2 & 2 & 0 & 10 \\
\hline infiltration & 1 & 16 & 1 & 0 & 0 & 0 & 1 & 0 & 2 \\
\hline Chorioamnionitis & $\mathrm{i}$ & 13 & $i$ & 0 & 1 & 0 & 1 & 0 & 0 \\
\hline
\end{tabular}


babies presenting with ophthalmia neonatorum. The table shows that $C$ trachomatis was recovered from the eyes of four babies. Chorioamnionitis had been observed in one of these deliveries and a granulocytic invasion of the membranes in another (case 1). None of the babies had a gonococcal eye infection, but potentially pathogenic bacteria were isolated from 12. Granulocystic invasion of the placental membrane was observed in only two of these 12 deliveries.

\section{Discussion}

We report the isolation of $N$ gonorrhoeae from three placentas at term and propose that, in at least the first two patients, the infection preceded rupture of the membranes.

Isolation of $N$ gonorrhoeae from the placenta would most logically result from one of three possible mechanisms: contamination of the placenta by passage through an infected birth canal, ascension of bacteria after rupture of the membrane, or prepartal infection of the placenta.

At parturition, the placenta can be expelled with the fetal side either folded in (the most common orientation) or folded out. In the latter case, the fetal side of the placenta would be heavily contaminated with vaginal bacteria. This was observed in 32 $(16.8 \%)$ of the deliveries studied. In the remainder, the placental culture was sterile or showed a monomicrobial population, as in the three patients with gonorrhoea that we described. In these cases, the fetal side of the placenta was probably folded in and the bacteriological status in utero maintained.

Most of the reported instances of orogastric contamination of newborn babies ${ }^{(6-9)}$ or gonococcal ophthalmia neonatorum after caesarean section $(1011)$ were complicated by rupture of the placental membranes 18 to 24 hours or more before birth, which allowed sufficient time for gonococci to ascend and establish an infection. Such premature rupture of membranes was not observed in the three cases presented here.

Ascension of bacteria after rupture of the membranes can be excluded in our first two patients, as both placentas had sufficient bacteria to be visible on direct microscopical examination. The first was heavily contaminated and even had intracellular diplococci. The second placenta had fewer diplococci on direct examination, but $N$ gonorrhoeae was isolated only from the placenta and not from the cervical swab. The third patient described could have had either a recent infection or ascension of bacteria after the membranes were ruptured because placental infection was very light, there was no inflamation of the placental membranes, and $N$ gonorrhoeae was also isolated from the cervical swab.

These infections did not give rise to postpartum fever in the mothers or their offspring, which is not surprising in the light of the common isolation of $N$ gonorrhoeae from asymptomatic women. ${ }^{5}$ Mild infections held in check naturally (for example, by components of the amniotic fluid) ${ }^{12}$ may be common, and possibly only exceptional cases lead to premature rupture of membranes or other disease.

We thank S Ossari, C Péquignot, and the midwives of the obstetrics department of the Franceville Provincial Hospital for their excellent technical help, A Béville for secretarial help, and G Béville for documentation research.

\section{References}

1. Goodrich JT. Treatment of gonorrhea in pregnancy. Sex Transm Dis 1979;6 suppl 2: 168-73.

2. Handsfield HH, Hodson WA, Holmes KK. Neonatal gonococcal infection. I. Orogastric contamination with Neisseria gonorrhoeae. JAMA 1973;225:697-701.

3. Edwards LE, Barrada MI, Hamann AA, Hakanson EY. Gonorrhea in pregnancy. Am J Obstet Gynecol 1978; 132: $637-41$

4. Yvert F, Riou JY, Frost E, Ivanoff B. Les infections gonococciques au Gabon (Haut-Ogooué). Pathol Biol (Paris) 1984; 32: 80-4.

5. Walter P, Garin Y, Blot P. Placental pathologic changes in malaria. A histologic and ultrastructural study. Am J Pathol 1982; 109:332-44.

6. Nickerson CW. Gonorrhea amnionitis. Obstet Gynecol 1973; 42:815-7.

7. Rotbard MN, Gregory T, Salerno LJ. Intrapartum gonococcal amnionitis. Am J Obstet Gynecol 1975; 121:565-6.

8. Carbonetto $\mathrm{C}$. Neonatal gonococcal orogastric contamination. JAMA 1978; 240:861.

9. Oppenheimer EH, Winn KJ. Fetal gonorrhea with deep tissue infection occurring in utero. Pediatrics 1982; 69: 74-6.

10. Thompson TR, Swanson RE, Wiesner PJ. Gonococcal ophthalmia neonatorum: relationship of time of infection to relevant control measures. JAMA 1974;228:186-8.

11. Strand CL, Arango VA. Gonococcal ophthalmia neonatorum after delivery by cesarean section: report of a case. Sex Transm Dis 1979; 6: 77-8.

12. Schlievert P, Larsen B, Johnson W Gazask RP. Bacterial growth inhibition by amniotic fluid. III. Demonstration of the variability of bacterial growth inhibition by amniotic fluid with a new plate-count technique. Am J Obstet Gvnecol 1975; 122:809-19. 